\title{
A New Urban Segregation-Growth Coupled Model using a Belief-Desire-Intention Possibilistic Framework
}

\author{
Meili Vanegas-Hernandez* \\ Universidad de los Andes, \\ Colombia \\ m.vanegas10@uniandes.edu.co \\ Giovanni Fusco \\ Université Côte d'Azur, CNRS, \\ ESPACE, France \\ giovanni.fusco@unice.fr
}

\author{
Célia da Costa Pereira \\ Université Côte d'Azur, CNRS, \\ I3S, France \\ celia.pereira@unice.fr \\ Andrea G. B. Tettamanzi \\ Université Côte d'Azur, CNRS, \\ I3S, France \\ andrea.tettamanzi@unice.fr \\ José Tiberio Hernández \\ Universidad de los Andes, \\ Colombia \\ jhernand@uniandes.edu.co
}

\author{
Diego Moreno \\ Université Côte d'Azur, CNRS, \\ ESPACE, France \\ diego.moreno@unice.fr \\ Michel Riveill \\ Université Côte d'Azur, CNRS, \\ I3S, France \\ michel.riveill@unice.fr
}

\begin{abstract}
We study the feasibility of using Belief, Desire and Intention agents for modeling the phenomena of urban growth and segregation. Uncertainty, typical of real world situations is modeled using possibility theory. We have also implemented a simple visualization tool whose aim is to track the changes in the model. Some preliminary experiments suggest that such an approach might allow a decision-maker to dynamically track the changes in the model. Besides, it is also possible to interact with the different steps of the simulation via the model. Our proposal makes it possible to simulate the interactions between cognitive agents in an economical environment while taking the spatial context into account.
\end{abstract}

\section{CCS CONCEPTS}

- Computing methodologies $\rightarrow$ Intelligent agents; Agent / discrete models; • Applied computing $\rightarrow$ Economics; Sociology;

\section{KEYWORDS}

Agent-Based Modeling, Geosimulation, BDI Agent Model, Urban Segregation

ACM Reference format:

Meili Vanegas-Hernandez, Célia da Costa Pereira, Diego Moreno, Giovanni Fusco, Andrea G. B. Tettamanzi, Michel Riveill, and José Tiberio Hernández. 2017. A New Urban Segregation-Growth Coupled Model using a Belief-Desire-Intention Possibilistic Framework.

\footnotetext{
*A part of the work described here was carried out while the author was visiting the Université Côte d'Azur.

WI '17, August 23-26, 2017, Leipzig, Germany

(C) 2017 Association for Computing Machinery.

This is the author's version of the work. It is posted here for your personal use. Not for redistribution. The definitive Version of Record was published in Proceedings of WI '17, August 23-26, 2017, https: //doi.org/10.1145/3106426.3106486.
}

In Proceedings of WI '17, Leipzig, Germany, August 23-26, 2017, 9 pages.

https://doi.org/10.1145/3106426.3106486

\section{INTRODUCTION}

Geosimulation tools are useful to analyze the impact that planning decisions may have on urban systems [5]. The Transport Oriented Modeling for urban denSification Analysis (TOMSA) project aims to create tools for urban planners to analyze evolution of urban forms and segregation patterns in fast changing cities. Usually, these two aspects of urban systems are analyzed separately [4]. As a first proposal, this work presents a new kind of agent-based model as a starting point for future research in urban modelling: adopting a possibilistic framework for rule generation of heterogeneous agents, coupling urban segregation and morphogenesis processes, integrating spatial constraints in agents behaviours. The paper is organized as follows. In Section 2, we present some related work in urban modelling and Agent Based Modelling (ABM). The description of the ABM framework used, a Belief-Desire-Intention (BDI) like architecture, is then presented in Section 3. Section 4 provides the details about the implementation and the experiments carried out in which we have considered simplified urban spaces in the real case of Bogota (Colombia). Finally, some conclusions and perspectives of future work are presented in Section 5.

\section{RELATED WORK}

In recent years, the complexity of cities has often been tackled by simulation tools that represent urban systems as multiple elements interacting locally between them and generating global patterns, like agent-based models (ABM) [3, 5].

A known theoretical representation of urban segregation using simple agents was proposed by Schelling in 1971 [16]. In Schelling's spatial proximity model, agents are divided in two groups. Initially, they are located randomly. At each step, 
agents evaluate their satisfaction based on the ratio between the number of agents of the same group and the number of agents in their neighbourhood. If the ratio is greater than a certain threshold, they decide to move to a random empty plot that satisfies them. Segregation patterns emerge regardless of the critical threshold value. The model has been widely analyzed and extended [6]: testing robustness of Schelling's results with respect to different definitions of individual utilities and/or environments $[11,15]$, interpreting results as the outcome of a coordination problem $[18,19]$, or studying the impact of heterogeneous agents and public policies [13], for example. Gilbert [12] pointed out that the Schelling's model of residential segregation made only assumptions about the motivations of individual households without considering the reasons why households might decide to move out of neighbourhoods where they are in a minority - when simulating social behaviors of humans, we should consider the social aspects as well as the cognitive aspects of the agents' behavior we would like to mimic. Another interesting work, among others, promoting the use of the cognitive aspects of the agents in the social simulations is the one proposed by Sun [17], who underlined the added value of combining cognitive and social aspects in the simulation tools. The use of the KISS ("Keep It Simple, Stupid") principle advocated by Axelrod [2] has then started to leave room, when appropriate, to the KIDS ("Keep It Descriptively Simple") principle. Adam et al. [1] have recently proposed an interesting survey advocating the advantages of complex and/or descriptive models for keeping the programming intuitive and allowing agents to adapt to the environment and being able to explain their behaviour. However, none among those works considered the possibilistic uncertainty in the agent's beliefs and desires which, in our opinion, would make even more realistic the results of the simulation for the following reasons: (i) an agent may have a partial vision based on a qualitative order of uncertainty - we may just infer that a world is more possible than another and no probability measure can be computed - about its environment; (ii) the agent's opinions can change in the light of new (partially truth) information and (iii) the agent's goals may change consequently.

This paper presents a study of the feasibility of using BDI agents for modeling the phenomena of urban growth and segregation. In this preliminary study, we consider three kinds of agents, namely households, investors, and promoters. The interaction between them and their environment generates the relocation of citizens over a city, taking into account different factors. This proposal is based on the traditional Schelling Model but differs of it in four important aspects. In the first place, the household satisfaction takes into account not only the characteristics of the agents around it but also the proximity to the transport network and to city facilities. Also, the agents do not relocate randomly, as they consider the same proximities described above to relocate in a new empty plot. Third, promoters and investors interact with households desires and within spatial constrains in order to transform undeveloped land in new dwelling opportunities

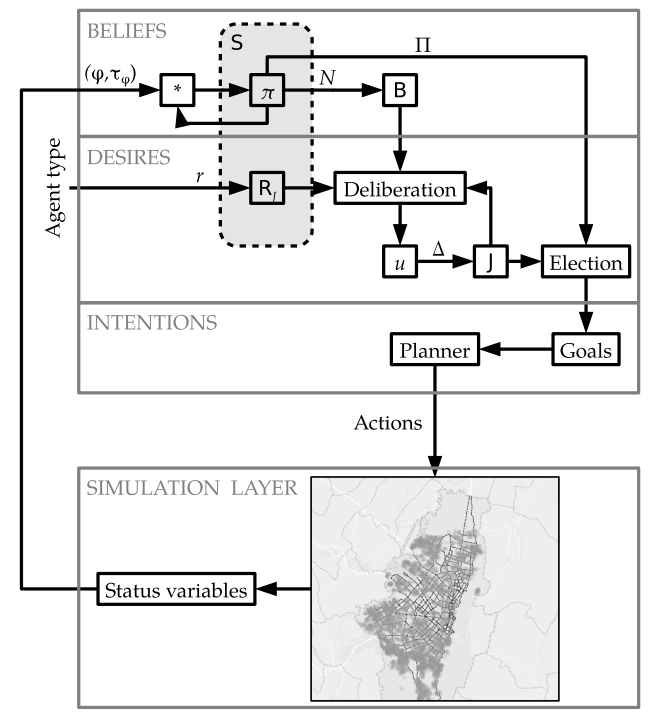

Figure 1: A Schema of the possibilistic BDI agentbased modeling framework.

for households. Possibilistic uncertainty is considered when modeling the cognitive aspects of the agents. The urban growth dynamics are thus coupled with the urban segregation dynamics. Some preliminaries experiments suggest that such an approach might allow a decision-maker to dynamically track the changes in the model.

\section{MODELING FRAMEWORK}

Figure 1 provides a schematic overview of the framework, which consists of two layers: the simulation layer, which contains the usual simulation mechanics, and the possibilistic BDI agent layer, which provides the reasoning and planning functionality of the cognitive agents involved in the model. This latter layer implements the possibilistic BDI model of agency proposed by da Costa Pereira and Tettamanzi [8].

An agent receives information from the simulation layer in the form of a formula $\varphi$ and its degree of certainty of $\tau_{\varphi}$ and thus forms its beliefs. The "program" of the agent consists of a number of desire-generation rules, contained in a rule-base $\mathcal{R}_{J}$. These, together with the beliefs, determine a set of goals, which are then fed into a planner module to compute the actions to be performed by the agent.

The internal mental state of the agent is completely described by a possibility distribution $\pi$ (from which the beliefs of the agent can be computed) and by a set of desiregeneration rules $\mathcal{R}_{J}$.

The set $\mathcal{J}$ of the agent's justified desires is generated dynamically through a deliberation process which applies the rules in $\mathcal{R}_{J}$ to the current beliefs and justified desires to produce a possibility measure $u$, which may be viewed as a qualitative utility function, associating to each possible world a degree to which it is desirable for the agent. 
Finally, the agent rationally elects its goals from the justified desires $\mathcal{J}$ as the most desirable of the possible sets of justified desires, according to its beliefs. The agent then plans its actions to achieve the elected goals.

For details about this model of agency, we refer the reader to [7-9]. Here we briefly recall few essential notions required to understand the proposed framework.

Information manipulated by a cognitive agent is represented symbolically by a classical propositional language.

Definition 1 (Language). Let $\mathcal{A}$ be a finite set of atomic propositions and let $\mathcal{L}$ be the propositional language such that $\mathcal{A} \cup\{\top, \perp\} \subseteq \mathcal{L}$. We have for all $\phi, \psi \in \mathcal{L}$, that: (i) $\neg \phi \in \mathcal{L}$, (ii) $\phi \wedge \psi \in \mathcal{L}$, (iii) $\phi \vee \psi \in \mathcal{L}$, and (iv) $\phi \supset \psi \equiv \neg \phi \vee \psi$.

A state (snapshot) of the world is represented by an interpretation which is a function associating a truth degree to each atomic proposition in $\mathcal{A}$. Formally, if we denote by $\Omega=\{0,1\}^{\mathcal{A}}$ the set of all interpretations on $\mathcal{A}$, an interpretation $\mathcal{I} \in \Omega$ is a function $\mathcal{I}: \mathcal{A} \rightarrow\{0,1\}$ assigning a truth value $p^{\mathcal{I}}$ to every atomic proposition $p \in \mathcal{A}$ and, by extension, a truth value $\phi^{\mathcal{I}}$ to all formulas $\phi \in \mathcal{L}$.

Definition 2. The notation $[\phi]$ denotes the set of all models (namely, interpretations satisfying $\phi$ ) of a formula $\phi \in \mathcal{L}$ :

$$
[\phi]=\{\mathcal{I} \in \Omega: \mathcal{I} \models \phi\} .
$$

3.0.1 Possibility Theory. Possibility theory is a theory of epistemic uncertainty whose basic notion is a possibility distribution $\pi: \Omega \rightarrow[0,1]$ which assigns to each possible world a degree of plausibility. A possibility distribution for which there exists a completely possible value $\left(\exists v_{0} ; \pi\left(v_{0}\right)=1\right)$ is said to be normalized.

Definition 3 (Possibility and Necessity Measures). A possibility distribution $\pi$ induces a possibility measure and its dual necessity measure, denoted by $\Pi$ and $N$ respectively. Both measures apply to the models of a formula $\phi \in \mathcal{L}$ and are defined as follows:

$$
\begin{aligned}
& \Pi([\phi])=\max _{\mathcal{I} \mid=\phi} \pi(\mathcal{I}) ; \\
& N([\phi])=1-\Pi([\neg \phi])=\min _{\mathcal{I} \mid \models \phi}\{1-\pi(\mathcal{I})\} .
\end{aligned}
$$

3.0.2 Representing Beliefs and Desires. The beliefs of an agent are represented by a normalized possibility distribution $\pi$, while its desires are represented by a possibility distribution $u$, which may be viewed as a qualitative utility function; $\pi(\mathcal{I})$ represents the plausibility order of the possible world situation represented by interpretation $\mathcal{I}$, whereas $u(\mathcal{I})$ represents how much world $\mathcal{I}$ would be desirable for the agent.

Such qualitative utility is determined by a set of desiregeneration rules, also taking the beliefs of the agent into account [7].

Definition 4 (Desire-Generation Rule). A desire-generation rule $R$ is an expression of the form $\beta_{R}, \psi_{R} \Rightarrow_{D}^{+} \phi$, where $\beta_{R}, \psi_{R}, \phi \in \mathcal{L}$. The unconditional counterpart of this rule is $\alpha \Rightarrow_{D}^{+} \phi$, with $\alpha \in(0,1]$.
The intended meaning of a conditional desire-generation rule is: "an agent desires every world in which $\phi$ is true at least as much as it believes $\beta_{R}$ and desires $\psi_{R}$ ", or, put in terms of qualitative utility, "the qualitative utility attached by the agent to every world satisfying $\phi$ is greater than, or equal to, the degree to which it believes $\beta_{R}$ and desires $\psi_{R}$ ". The intended meaning of an unconditional rule is that the qualitative utility of every world $\mathcal{I} \models \phi$ is at least $\alpha$ for the agent.

The degree to which the agent believes a formula $\phi$ is given by the necessity $N([\phi])$ induced by $\pi$.

The possibility distribution $\pi$ together with the desiregeneration rules define what may be called the mental state of an agent.

Three types of agents are defined in order to simulate the evolution of urban forms and segregation patterns in a city. First, the households, that relocate over the city taking into account their beliefs and desires. Second, the promoters, that build properties all over the city, trying to build in areas in which households are interested to buy, in order to increase sales. Lastly, the investors, that buy properties to rent them to other households. A household agent is defined as an object with a given purchasing power and a net monthly income. Similar to the household, the promoters and the investors are objects with a purchasing power, but in this case, net monthly income is not contemplated. This characteristic was defined considering that promoters and investors are also households. This mechanism allows a promoter agent to have the same purchasing power as its corresponding household agent. When the promoter's goals are related to build, it acts as a promoter agent, however, when its goals are focused in buying or renting a house for its household, it acts as a household.

Each agent has also some behavioral parameters that affect its decision making process. For example, a household has an investing degree, that determines the susceptibility to become an investor. On the other hand, an investor has a speculation degree, that establishes whether the agent is inclined to speculate or not. Lastly, a promoter has a risk aversion degree, which indicates how likely it is for the agent to take financial risks. Most of these parameters where assigned randomly for every agent in the simulation process.

Furthermore, a city is defined as the union of a transport network, a set of equipments of different kinds, a delimited number of urban plot lands, and a set of clear administrative divisions within the city (i.e. neighborhoods). These parameters can be established before the simulation starts. The simulation interacts with each parameter in a syntactic level, without considering specific characteristics of each one of them.

\section{IMPLEMENTATION, EXPERIMENTS AND RESULTS}

The behavior of the three types of agents in the model is described by the following desire-generation rules, which allow the agents to change their desires (and, possibly, perform 
Table 1: Meanings of the propositional symbols of $\mathcal{A}$.

\begin{tabular}{|c|l|}
\hline Symbol & Meaning \\
\hline$a b$ & afford buying property \\
$a b l$ & afford buying land \\
$a c$ & afford constructing \\
$a r$ & afford renting \\
$b$ & buy a property \\
$b r$ & buying would be profitable \\
$c h$ & change \\
$d i s$ & be dissatisfied \\
$i$ & invest \\
$l$ & be a landlord \\
$o$ & be an owner \\
$r$ & rent \\
$r a$ & be risk averse \\
$s$ & sell \\
$s o p$ & sell off plans \\
$s p$ & speculate \\
$s r$ & selling would be profitable \\
\hline
\end{tabular}

actions as a consequence) in response to changes in the simulation layer. Table 1 lists the propositional symbols used in the BDI agent layer.

\section{Desire generation rules for Household Agent}

(1) The investing degree for the current household is $\alpha$ :

$$
\alpha \Rightarrow_{D}^{+} i
$$

(2) If the household does not desire to invest, the household does not desire to become a landlord:

$$
\top, \neg i \Rightarrow_{D}^{+} \neg l
$$

(3) If the household believes it affords buying and the household desires to change then the household desires to buy:

$$
a b, c h \Rightarrow_{D}^{+} b
$$

(4) If the household desires to change and believes it does not afford buying but believes it affords renting then the household desires to rent:

$$
\neg a b \wedge a r, c h \Rightarrow_{D}^{+} r
$$

(5) If the household believes it is owner and it is dissatisfied, then the household desires to change and either sell or become an investor:

$$
o \wedge d i s, \top \Rightarrow_{D}^{+} \operatorname{ch} \wedge(s \vee l)
$$

(6) If the household believes it affords buying, has buying profitability and desires to invest, then the household desires to buy and become an investor:

$$
a b \wedge b r, i \Rightarrow_{D}^{+} b \wedge l
$$

\section{Desire generation rules for Investor Agent}

(1) The investing degree of the current investor is $\beta$ :

$$
\beta \Rightarrow{ }_{D}^{+} i
$$

(2) If the investor believes that it is owner and, in the current state, selling is more profitable than continuing with the current property as a landlord and desires not to speculate, then the investor desires selling:

$$
o \wedge s r, \neg s p \Rightarrow_{D}^{+} s
$$

(3) If the investor believes that it is owner and, in the current state, continuing with the current property is more profitable than selling, then the investor decides not to sell:

$$
o, s p \Rightarrow \stackrel{+}{D} \neg s
$$

(4) If the investor believes it affords buying and has buying profitability and desires to invest, then the investor desires to buy and create a new propertyinvestor relation:

$$
a b \wedge b r, i \Rightarrow_{D}^{+} b \wedge l
$$

\section{Desire generation rules for Promoter Agent}

(1) The risk aversion degree of the current promoter is $\gamma:$

$$
\gamma \Rightarrow_{D}^{+} r a
$$

(2) If the promoter believes it affords to buy the land and doesn't afford to construct and desires not to be risk averse, then it desires to buy the land and sell off plans:

$$
a b l \wedge \neg a c, \neg r a \Rightarrow_{D}^{+} b l \wedge s o p
$$

\subsection{Simulation Layer}

The model involves five distinct types of objects: land plots, properties, promoters, investors, and households. During a simulation step, each one of these classes is updated and each object from the different kinds is updated synchronously. More precisely, when a first household has been updated, the updating process concerning another household has to consider the state of the world which results from the changes due to the decisions made by the first household.

The simulation starts by updating the land plots. A land plot has a unique variable characteristic over time-its price. This value was assigned randomly to each of the 1000 plots proposed at the beginning of the simulation. Properties follow O'Sullivan's well-known gentrification model [14], which describes the behavior of the price, capitalized rent, potential rent and value of a property. O'Sullivan defined each of these characteristics as follows: a property's value is the sum of the materials and labor used to construct it; the capitalized rent corresponds to the income the owner receives for renting the property in a specific time $t$ and the potential rent is the rent the owner might receive if the property were in its finest shape at a specific time $t$; finally, the price is calculated as the sum the property's value and capitalized rent

$$
\operatorname{price}(t)=\text { value }(t)+\text { capitalized_rent }(t) \text {. }
$$

where the value follows the depreciation law

$$
\operatorname{value}(t)=0.97 \cdot \operatorname{value}(t-1) \text {. }
$$


The initial values for each of the property's characteristics were assigned randomly, in this case, the price, the value and an original rent. The potential and capitalized rent derive from the original rent, as they have the same value at the beginning of the simulation. Also, the value is assigned as a percentage of the price. This percentage may vary between $30 \%$ and $90 \%$ of the property's price.

After the land plots and properties are updated, the promoters' characteristics are updated too. In this case, the object in consideration is associated to an agent, which changes its set of beliefs and desires over time, taking into account the status of the simulation. Each belief depends on some characteristics of the agent and of its environment. In this case, the promoter has a purchasing power $W$, whose initial value is assigned randomly. This value evolves in time, according to the following equation:

$$
W(t)= \begin{cases}W(t-1), & \text { if Rnd }<0.6 ; \\ 1.03 \cdot W(t-1), & \text { if } 0.6 \leq \text { Rnd }<0.8 \\ 0.97 \cdot W(t-1), & \text { otherwise. }\end{cases}
$$

Purchasing power of promoters remains thus stable most of the times, but mild variations can occur, due to real-estate market fluctuations: in $20 \%$ of the cases variation is positive and in $20 \%$ of the cases, it is negative. The last characteristic of a promoter, risk aversion $\gamma$, is initialized randomly for each promoter agent.

A promoter agent updates its beliefs with information taken from the simulation, namely afford buying land $(a b l)$ and afford constructing $(a c)$. The former tells how strongly the promoter believes it can afford to buy a plot of land, while the latter how strongly the promoter believes it can afford to construct.

In order to update the degree a promoter believes $a b l$, every land plot's price is evaluated and compared to the promoter's purchasing power. The degree of belief is proportional to the fraction of land plots the promoter can afford: $\forall j \in P$,

$$
\tau_{a b l}(j)=\frac{1}{n} \sum_{i=0}^{n-1} x_{i},
$$

where $P$ is the set that contains all the promoters, $n$ is the number of available lands, and

$$
x_{i}= \begin{cases}1, & \text { if } W_{j}>\text { price }_{i} \\ 0, & \text { otherwise }\end{cases}
$$

The $a c$ belief is updated randomly at each time step.

The process of updating each promoter's beliefs is followed by updating the investors' beliefs. Some of the calculations are similar to the ones for the promoter. For example, the desires to speculate $s p$ and to invest $i$. Both of them are desires, not beliefs. They former tells how much an investor agent desires to speculate, in other words, how desirable it is for the agent to buy hoping the value of the acquired property would increase in the future. Both $s p$ and $i$ are updated randomly.

On the other hand, the investor has an afford buying belief $(a b)$, which is calculated like the $a b l$ belief of the promoter agent. Also, if an investor is associated to a household, at each time step, the household's purchasing power and income are updated taking into account the capitalized rent of the investor's property.

In addition, the investor agent has a belief which evaluates if buying would be profitable (br). The degree of this belief is calculated as follows: $\forall j \in I$,

$$
\tau_{b r}(j)=\left\{\begin{array}{l}
\frac{\text { capitalizedRent }_{C P}}{\text { potentialRent }_{C P}} \\
\text { if } \text { price }_{C P}<W_{j} \\
0, \quad \\
\quad \text { otherwise }
\end{array}\right.
$$

where $I$ is the set of investors and $C P$ is the cheapest property in the market.

An investor might have desired to sell its property, if the property were not profitable. This desire could be generated if selling that property were profitable $(s r)$, whose degree of belief is calculated as follows: $\forall j \in I$,

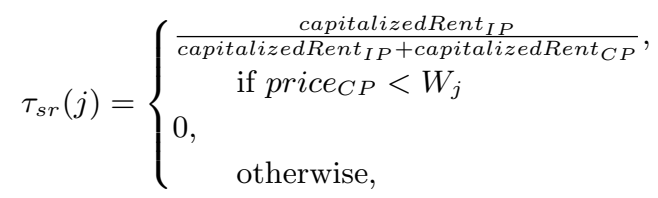

where $I$ is the set of investors, $I P$ is the investor's property, and $C P$ is the cheapest property in the market at a specific time step $t$. It is important to highlight that an investor entity owns 0 or 1 properties. If an investor wants to buy more properties, a new investor entity is generated and associated to it. This information feeds the is owner belief $(o)$, which is true if the investor owns a property and false otherwise.

Finally, the households are updated. Some of the household's characteristics are very similar to the other agents' characteristics. An example is the household's purchasing power. The behavior of this characteristic is like for the promoter agent. An important difference between a household and an investor or promoter is the fact that a household has a net monthly income $N M I$, which varies over time, according to the following law:

$$
N M I(t)= \begin{cases}N M I(t-1), & \text { if Rnd }<0.6 ; \\ 1.03 \cdot N M I(t-1), & \text { if } 0.6 \leq \mathrm{Rnd}<0.8 \\ 0.97 \cdot N M I(t-1), & \text { otherwise. }\end{cases}
$$

A household may desire to move out; this desire is called ch, for changing. Also, a household can become an investor if they bought a property for renting. This decision is governed by the $i$ desire. Both $c h$ and $i$ are randomly generated.

Another belief generated for the household agent is dissatisfaction (dis), whose degree is given by $\tau_{d i s}=2^{-V}$, where $V$ is the overall value of its current property, given by, $\forall i \in H$,

$$
V(t, i)=\kappa_{q} \times V_{q}+\kappa_{c} \times V_{c}+\kappa_{p} \times V_{p},
$$

where $V_{q}$ is the quality value, $V_{c}$ the centrality value, and $V_{p}$ is the proximity value, $H$ is the set that contains all the households and $t$ is the step in the simulation, or time. The definition of each of the coefficients $\kappa_{q}, \kappa_{c}$, and $\kappa_{p}$ depends 
on the case study. In Section 4.2, each of the case studies will have different coefficients.

Each of these coefficients is assigned randomly when a household agent is created and is treated as immutable, so that every household has different inclinations. For example, one household could prefer to have a higher value in proximity than centrality, another could prefer the opposite. On the other hand, each of the values are calculated taking into account the agent's environment.

One of the purposes of the simulation is to allow the urban planner to analyze changes between steps of the simulation in the households distribution over the city. In order to provide useful information to contribute to the analysis, some indicators were defined along with the urban expert. The first indicator is the ratio of tenant households and landowners in an administrative division and in the city as a whole,

$$
R O P=\frac{n R}{n O},
$$

where $n R$ is the number of rented properties, $n O$ is the number of properties occupied by the landowner. This indicator is calculated for all the city at every step of the simulation.

The second indicator is a segregation index proposed by Duncan \& Duncan [10]:

$$
S I_{i}=\frac{\left|\frac{n R_{i}}{n R T}-\frac{n O_{i}}{n O T}\right|}{2}, \quad \forall i \in D,
$$

where $n R_{i}$ is the number of rented properties in the urban division $i, n O_{i}$ is the number of properties occupied by the landowner in the urban division $i, n R T$ is the number of rented properties in the city, $n O T$ is the number of properties occupied by the landowner in the whole city $D$ is the set of urban divisions, in other words, $D=\{0$, numberOfUrbanDivisions$1\}$.

\subsection{Experiments and results}

4.2.1 Case Study: Schelling. There were considered two different scenarios to see the model's behavior as a whole, one in a theoretical space and another one in a real space. Both scenarios are described below.

The first case simulated a simple relocation of households, following some standards proposed by Schelling in 1971 [16]. In this scenario, the unique agent used is the households. The spatial data consists of a grid, occupied for $70 \%$ by agents of two different colors. The initial number of households of either color is the same and the agents are positioned randomly.

This case was tested in three different scenarios, but all of them considered the basic characteristics described above. In every scenario, the simulation stopped as soon as the results of the previous step were the same as the current state.

In this case study, each cell represents a property. The initial state of the simulation consisted in $70 \%$ of the cells taken by households, the rest of the cells represented available properties. In each step of the simulation, a household evaluated its current state, taking into account the beliefs and desires described above. If the agent decided to move, it evaluated all the available properties and moved to one it could purchase and satisfied it the most. The satisfaction function varied in every scenario, as shown below.

- First Scenario: The satisfaction function takes into account the proximity to agents of the same kind. Equation 19 becomes here

$$
V(t, i)=0 \cdot V_{q}+0 \cdot V_{c}+1 \cdot V_{p},
$$

where, $\forall i \in H$,

$$
V_{p}(i, t)=\frac{1}{\|H\|} \sum_{j \in H} x_{i, j}
$$

and

$$
x_{i, j}= \begin{cases}1, & \text { if } d(i, j)<1 \mathrm{~km} \\ 0, & \text { otherwise }\end{cases}
$$

where $d(\cdot, \cdot)$ refers to the simplification of the Cartesian distance using 4326 as the SRID (Spatial Reference Identifier) and $H$ is the set of all the households.

- Second Scenario: The satisfaction function took into account the proximity to the transport network. Equation 19 becomes here

$$
V(t, i)=1 \cdot V_{q}+0 \cdot V_{c}+0 \cdot V_{p},
$$

where, $\forall i \in H$,

$$
V_{q}(i, t)=\frac{1}{\|N\|} \sum_{j \in N} x_{i, j},
$$

where $x_{i, j}$ is calculated as in Equation 24, $H$ is the set of households, and $N$ is the set of stretches in the transport network.

- Third Scenario: The satisfaction function took into account the proximity to agents of the same kind and transport network. Equation 19 becomes here

$$
V(t, i)=\frac{1}{2} V_{q}+0 \cdot V_{c}+\frac{1}{2} V_{p}
$$

where $V_{q}$ and $V_{p}$ are calculated as in Equations 23 and 26 .

The first scenario represented the Schelling's Model using the proposed framework. Figure 2 shows the initial and the final step of the simulation of the Schelling's Model using the proposed framework. There were two different groups of household agents, blue and red and their initial distribution is assigned randomly.

The results, after the simulation process was over, are presented in Figure 2b. The agents seemed to be segregated all over the grid in the blue and red groups.

The second one introduced a testing transport network. In this case, when an agent decided to relocate, it did not relocate randomly. It had associated an utility function that considered the closeness to the transport network. The agent always tried to maximize his utility function when relocating to an available spot.

In this case, the agents turned to be closer to the transport network in the last iteration of the simulation.

At last, the utility function calculated by each agent when relocating to a new spot also considered the closeness to similar agents. The results are presented in Figure 4 . 


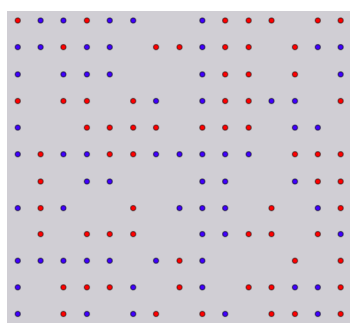

(a)

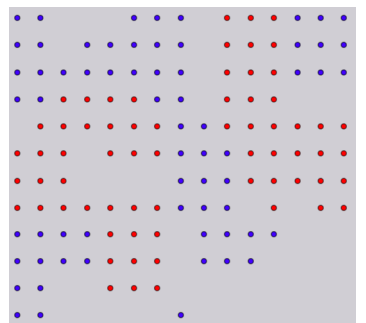

(b)
Figure 2: (a) The location of the households in $t=0$. (b) The location of the households in $t=9$ for the simulation of the Schelling's Model using the proposed framework in the theoretical case.

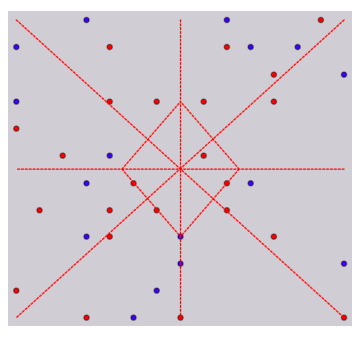

(a)

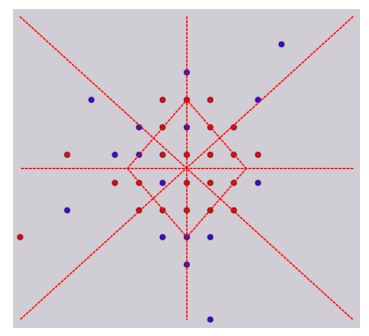

(b)
Figure 3: (a) The location of the households in $t=0$. (b) The location of the households in $t=9$ for the scenario with a testing transport network using the framework in the theoretical case.

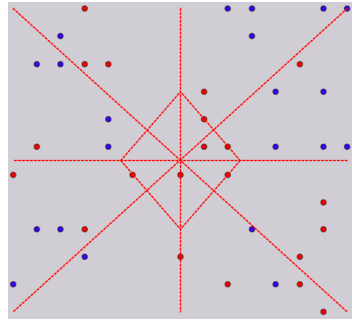

(a)

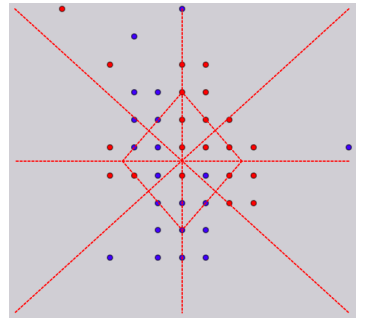

(b)
Figure 4: (a) The location of the households in $t=0$. (b) The location of the households in $t=9$ for scenario with a testing transport network and a closeness requirement in the theoretical case.

In the last case, the agents grouped all over the transport network at the same time they segregated in the two groups proposed, as shown in Figure 4b.

4.2.2 Case Study: Bogota. The second case was geographically located in Bogota, Colombia. In the initial state of the world, there were 100 agents of each type with random behavioral degrees. In the initial state, there were no constructed properties, but there were 1000 land plots available to construct, distributed all over the city. Also, the simulation considered a real transport network with information of 234 tranches in Bogota, and over 5000 of equipments distributed all over the city.

This case was also tested in multiple scenarios, trying to map the scenarios in the theoretical case to the ones in the real case. In each step of the simulation, of every scenario, a household evaluated its current state, taking into account the beliefs and desires described in the previous subsection. If the agent decided to move, it evaluated all the available properties and moved to one it could purchase and satisfied it the most. The satisfaction function varied in every scenario, as shown below.

- First Scenario: The satisfaction function was calculated randomly. Equation 19 becomes

$$
V(t, i)=\frac{1}{3} V_{q}+\frac{1}{3} V_{c}+\frac{1}{3} V_{p} .
$$

In this case, all the values, $V_{q}, V_{c}$ and $V_{p}$ are random numbers.

- Second Scenario: The satisfaction function took into account the proximity to a unique route in the transport network. Equation 19 becomes

$$
V(t, i)=1 \cdot V_{q}+0 \cdot V_{c}+0 \cdot V_{p},
$$

where, $\forall i \in H$,

$$
V_{q}(i, t)=\frac{1}{\|N\|} \sum_{j \in N} x_{i, j},
$$

where $x_{i, j}$ is calculated as in Equation $24, H$ is the set of households, and $N$ is the set of stretches in the transport route considered.

- Third Scenario: The satisfaction function took into account the proximity to a unique route in the transport network and a set of equipments in the city. Equation 19 becomes

$$
V(t, i)=1 \cdot V_{q}+0 \cdot V_{c}+0 \cdot V_{p},
$$

where, $\forall i \in H$,

$$
V_{q}(i, t)=\frac{1}{2\|S\|} \sum_{j \in S} x_{i, j}+\frac{1}{2\|E\|} \sum_{j \in E} x_{i, j},
$$

where $x_{i, j}$ is calculated as in Equation 24, $S$ is the set of stretches in the transport route and $E$ is the set of equipments considered.

- Fourth Scenario: The satisfaction function took into account the proximity to the entire transport network and the entire set of equipments of the city. Equation 19 becomes

$$
V(t, i)=1 \cdot V_{q}+0 \cdot V_{c}+0 \cdot V_{p},
$$

where, $V_{q}$ is calculated as in Equation 32, but here $S$ is the number of stretches of the entire transportation network and $E$ is the set of all the equipments.

In the first scenario, the agents had a satisfaction function with which they evaluated their current state. When that satisfaction function was lower than a specific threshold, in 
this case, 0.5 , the agent decided to relocate to a new random available spot. The results are shown in Figure 5.

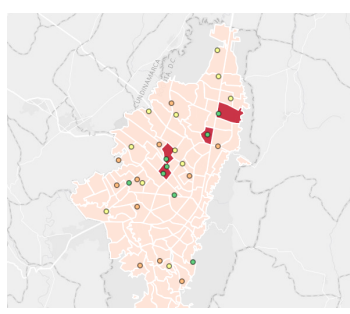

(a)

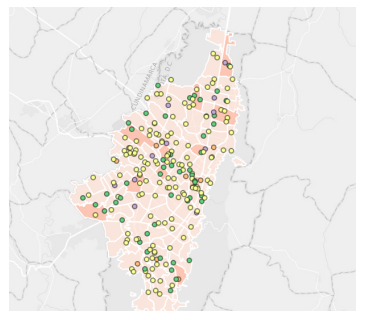

(b)
Figure 5: (a) The location of the households in $t=0$. (b) The location of the households in $t=9$ for the scenario in which agents relocate randomly in the real case.

The points in the map represent the households. Figure $5 \mathrm{~b}$ shows a random distribution of the agents all over the city, with no pattern detected.

The next scenario introduces one artery of the transport network, and the agents evaluate the closeness to this artery when they decide to relocate. It is important to take into account that the households are classified by income in different groups, and each group is represented by different colors.

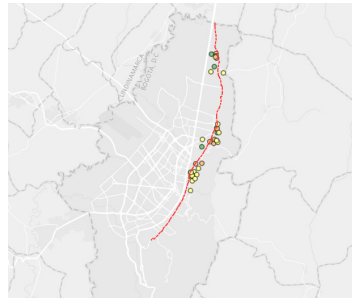

(a)

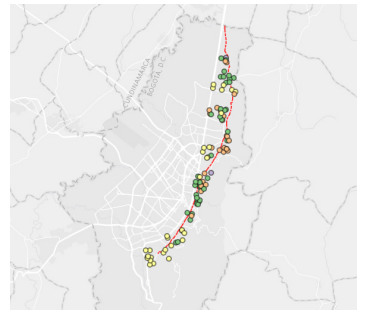

(b)
Figure 6: (a) The location of the households in $t=0$. (b) The location of the households in $t=9$ for the case of one artery of the transport network in the real case.

The results presented in Figure 6 shows how the agents are grouped all over the artery.

The third scenario introduces a plot in the city with some equipments. In this case, the weight of being close to the transport network and the weight of being close to a city equipment is the same for the agent's satisfaction function. The plot in the city with city equipments is represented as a blue pixel.

The results in Figure 7 present how a particular group of the population ends up near to the blue spot and the remaining group ends up near to the transport network artery. Figure 8 considers all the actual equipments in the city of Bogota and the principal arteries in the transport network. In this scenario, the agents act exactly as the ones presented in Figure 7 above.

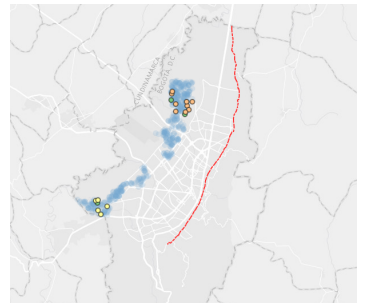

(a)

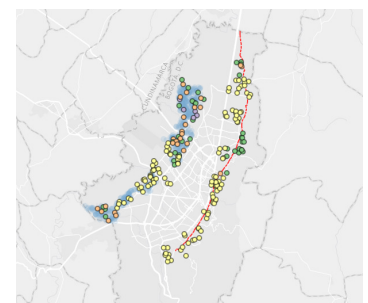

(b)
Figure 7: (a) The location of the households in $t=0$. (b) The location of the households in $t=9$ for the third scenario in the second case

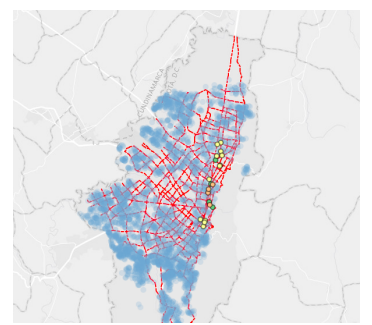

(a)

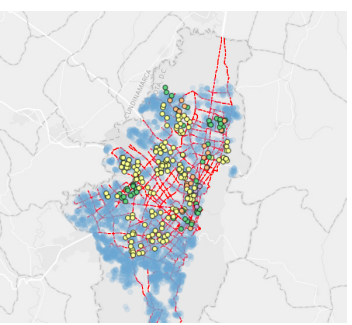

(b)
Figure 8: (a) The location of the households in $t=0$. (b) The location of the households in $t=9$ for scenario with all the equipments and the entire transport network in the real case.

\section{CONCLUSIONS AND FUTURE WORK}

We introduced a scalable framework that allows to represent households, investors, and promoters, while using possibilistic BDI agents, interacting over a spatial context as a segregation model. The framework addresses multiple characteristics in a qualitative fashion, including some assumptions considered in the agents definition and the simple definition and implementation of time in the simulation process. Achieving a high accuracy of results was not the goal of our study. Instead, the final objective this proposal pursues is a clear qualitative representation of the agents in a city, that visualizes the effects of new equipments or arteries in its transport network.

Although the satisfaction function of Equation 19 considers multiple characteristics of the agent and its environment, the case studies did not implement the entire definition. This is left for future work.

The first experiments, conducted both with simplified and real case urban environments, seemed able to generate a variety of spatial patterns in agreement with more classic models not using a BDI framework. The coupling of the segregation sub-model with the urban growth sub-model remains to be further exploited.

In another direction, the model could be further developed to better take into account social dynamics within the city. We 
could for example implement the effect of social hierarchies on agent's satisfaction (owners being dissatisfied when too many renters live around them, but not the reverse). We could also model the formation of collectively shared beliefs within social groups. Collective beliefs could then be used as default, defeasible values for individual beliefs.

\section{Acknowledgments}

The work described in this paper was partially supported by the ECOS-Nord TOMSA (Transport Oriented Modeling for urban denSification Analysis) Project.

\section{REFERENCES}

[1] C. Adam and B. Gaudou. 2016. BDI agents in social simulations: a survey. Knowledge Eng. Review 31, 3 (2016), 207-238.

[2] R. Axelrod. 1997. The Complexity of Cooperation: Agent-Based Models of Competition and Collaboration. Princeton U. Press.

[3] M. Batty. 2007. Cities and complexity: understanding cities with cellular automata, agent-based models, and fractals. The MIT press.

[4] B. Bellman. 2014. Segregation and Urban Form: Towards an Understanding of Dynamics Between Race, Population Movement, and the Built Environment of American Cities. (2014).

[5] I. Benenson and P. Torrens. 2004. Geosimulation: Automatabased modeling of urban phenomena. John Wiley \& Sons.

[6] W. Clark and M. Fossett. 2008. Understanding the social context of the Schelling segregation model. PNAS 105, 11 (2008), 41094114.

[7] C. da Costa Pereira and A. Tettamanzi. 2010. Belief-Goal Relationships in Possibilistic Goal Generation. In ECAI 2010. 641646.

[8] C. da Costa Pereira and A. Tettamanzi. 2010. An Integrated Possibilistic Framework for Goal Generation in Cognitive Agents. In $A A M A S$ '10. 1239-1246.

[9] C. da Costa Pereira and A. Tettamanzi. 2014. Syntactic Possibilistic Goal Generation. In ECAI'14. 711-716.

[10] O. Duncan and B. Duncan. 1955. A Methodological Analysis of Segregation Indexes. American Sociological Review 20, 2 (1955), 210-217.

[11] G. Fagiolo, M. Valente, and N. Vriend. 2007. Segregation in networks. Journal of Economic Behavior \& Organization 64 , 3-4 (2007), 316-336.

[12] N. Gilbert. 2006. When does social simulation need cognitive models? In Cognition and Multi-Agent Interaction: From Cognitive Modeling to Social Simulation, R. Sun (Ed.). Cambridge University Press, Cambridge, 428-432.

[13] A. O'Sullivan. 2009. Schelling's model revisited: Residential sorting with competitive bidding for land. Regional Science and Urban Economics 39, 4 (2009), 397-408.

[14] D. O'Sullivan. 2002. Toward micro-scale spatial modeling of gentrification. Journal of Geographical Systems 4, 3 (2002), 251-274.

[15] R. Pancs and N. Vriend. 2007. Schelling's spatial proximity model of segregation revisited. Journal of Public Economics 91, 1-2 (2007), 1-24.

[16] T. Schelling. 1971. Dynamic models of segregation. The Journal of Mathematical Sociology 1, 2 (1971), 143-186.

[17] R. Sun. 2006. Prolegomena to integrating cognitive modeling and social simulation. Cambridge University Press. 3-28 pages.

[18] J. Zhang. 2004. A dynamic model of residential segregation. The Journal of Mathematical Sociology 28 (2004), 147-170.

[19] J. Zhang. 2004. Residential segregation in an all-integrationist world. Journal of Economic Behavior and Organization 54 (2004), 533- 550 . 